\title{
Stability in Generalized Modified Gravity
}

\author{
S. Zerbini \\ Department of Physics, University of Trento, and \\ Gruppo Collegato INFN di Trento, Sezione di Padova, Italy
}

\begin{abstract}
The stability issue of a large class of modified gravitational models is discussed with particular emphasis to de Sitter solutions. Three approaches are briefly presented and the generalization to more general cases is mentioned.
\end{abstract}

Keywords: modified gravity, stability

PACS: 11.25.-w, 95.36.+x,98.80.-k

\section{INTRODUCTION}

It is well known that recent astrophysical data are in agreement with a universe in current phase of accelerated expansion, in contrast with the predictions of Einstein gravity in FRW space-time. It seems that the most part of energy contents (roughly 75\%) in the universe is due to mysterious entity with negative pressure: the so dubbed Dark Energy. The simplest explanation is $\Lambda \mathrm{CDM}$ : Einstein gravity plus a small positive cosmological constant suffers from the coincidence problem and the cosmological constant issue: $\Lambda_{o b} / \Lambda_{t h} \simeq 10^{-120}$, while if we assume supersymmetry, one has $\Lambda_{o b} / \Lambda_{t h} \simeq 10^{-60}$. In this case $w_{d e}=\frac{p_{d e}}{\rho_{d e}}=-1$. There exist alternative explanations. Among many, we recall:

i. Modification of gravity on large scale: DGP brane-world model [1].

ii. Dark energy associated with cosmological scalar fields, quintessence if $w_{d e}>$ -1 , phantom matter if $w_{d e}<-1$.

iii. Modified gravity models: $R \longrightarrow R+f(R)$.

All these models look like $\Lambda \mathrm{CDM}$, but with an effective non constant cosmological term.

\section{MODIFIED GRAVITY AS MODELS FOR DARK ENERGY}

The $\Lambda$ CDM model is the simplest possibility but, it is worth investigating more general modifications, possible motivations run from quantum corrections to string models: (for a recent review see [2] and references therein). We shall consider the modification of the kind $F(R)=R+f(R)$. Models of this kind are not new and they have been used in the past by many authors, for example as models for inflation, $f(R)=a R^{2}$ [3]. Recently their interest in cosmology was triggered by the model $f(R)=-\mu^{4} / R$, proposed in order to describe the current acceleration of the observable universe [4].

It is important to stress that these $F(R)$ models are conformally equivalent to Einstein's gravity, coupled with a self-interacting scalar field, Einstein frame formulation. We will consider only the Jordan frame, in which the dynamics of gravity is described 
by $F(R)$ with minimally coupled matter. Observations are typically interpreted in this Jordan frame.

Finally, we would like to mention the so called viable $F(R)$ models, which have recently been proposed [5], with the aim to describe the current acceleration with a suitable choice of $F(R)=R+f(R)$, but also to be compatible with local stringent gravitational tests of Einstein gravity $F(R)=R$. The main idea is the so called disappearing of cosmological constant for low curvature, and mimicking the $\Lambda$ CDM model for high curvature. Thus, the requirements are:

a. $f(R) \rightarrow 0, \quad R \rightarrow 0$, compatibility with local tests.

b. $f(R) \rightarrow-2 \Lambda_{0}, \quad R \rightarrow+\infty$, description of current acceleration.

c. Local stability of the matter.

As a illustration, we recall a recent example of viable model [6]

$$
f(R)=-\alpha\left(\tanh \left(\frac{b\left(R-R_{0}\right)}{2}\right)+\tanh \left(\frac{b R_{0}}{2}\right)\right)
$$

where $R_{0}$, and $\Lambda_{0}$ are suitable constants. Its advantages are a better formulation in the Einstein frame and a generalization that may also include the inflation era.

\section{THE DE SITTER STABILITY ISSUE}

The stability of the de Sitter solution, relevant for Dark energy, may be investigated in these $F(R)$ models in several ways. We limit ourselves to the following three approaches:

i. Perturbation of Esq. of motion in the Jordan frame.

ii. One-loop gravity calculation around de Sitter background.

iii. Dynamical system approach in FRW space-time.

We shall briefly discuss these three approaches. We may anticipate that the third one can easily be extended to more general modified gravitational models.

\section{Stability of $F(R)$ model in the Jordan frame}

The starting point is the trace of the equations of motion, which is trivial in Einstein gravity $R=-\kappa^{2} T$, but, for a general $F(R)$ model, reads

$$
3 \nabla^{2} f^{\prime}(R)-2 f(R)+R f^{\prime}(R)-R=\kappa^{2} T .
$$

The new non trivial extra degree of freedom is the Scalaron : $1+f^{\prime}(R)=e^{-\chi}$. Requiring $R=R_{0}=C S T$, one has de Sitter existence condition in vacuum

$$
R_{0}+2 f\left(R_{0}\right)-R_{0} f^{\prime}\left(R_{0}\right)=0 .
$$

Perturbing around $\mathrm{dS}: R=R_{0}+\delta R$, with $\delta R=-\frac{1+f^{\prime}\left(R_{0}\right)}{f^{\prime \prime}\left(R_{0}\right)} \delta \chi$, one arrives at Scalaron perturbation Eq.

$$
\nabla^{2} \delta \chi-M^{2} \delta \chi=-\frac{\kappa^{2}}{6\left(1+f^{\prime}\left(R_{0}\right)\right)} T
$$


One may read off the Scalaron effective mass

$$
M^{2} \equiv \frac{1}{3}\left(\frac{1+f^{\prime}\left(R_{0}\right)}{f^{\prime \prime}\left(R_{0}\right)}-R_{0}\right) .
$$

Thus, if $M^{2}>0$, one has stability of the $\mathrm{dS}$ solution and the related condition reads

$$
\frac{1+f^{\prime}\left(R_{0}\right)}{R_{0} f^{\prime \prime}\left(R_{0}\right)}>1 .
$$

If $M^{2}<0$, there is a tachyon and instability. Furthermore, one may show that $M^{2}$ has to be very large in order to pass both the local and the astronomical tests and $1+f^{\prime}(R)>0$, in order to have a positive effective Newton constant. The same result has been obtained within a different more general perturbation approach in [7].

\section{One-loop $F(R)$ quantum gravity partition function}

Here we present the generalization to the modified gravitational case of the study of Fradkin and Tseytlin [8], concerning Einstein gravity on dS space. One works in the Euclidean path integral formulation, with dS existence condition $2 F_{0}=R_{0} F_{0}^{\prime}$, assumed to be satisfied. The small fluctuations around this $\mathrm{dS}$ instanton may be written as

$$
g_{i j}=g_{(0) i j}+h_{i j}, \quad g^{i j}=g_{(0)}^{i j}-h^{i j}+h^{i k} h_{k}^{j}+\mathscr{O}\left(h^{3}\right), \quad h=g_{(0)}^{i j} h_{i j} .
$$

Making use of the standard expansion of the tensor field $h_{i j}$ in irreducible components, and making an expansion up to second order in all the fields, one arrives at a very complicated Lagrangian density $L_{2}$, not reported here, describing Gaussian fluctuations around $\mathrm{dS}$ space. As usual, in order to quantise the model described by $L_{2}$, one has to add gauge fixing and ghost contributions. Then, the computation of Euclidean one-loop partition function reduces to the computations of functional determinants. These functional determinants are divergent and may be regularized by the well known zeta-function regularization. The evaluation requires a complicated calculation [9] and, neglecting the so called multiplicative anomaly, potentially present in zeta-function regularized determinants (see [10]), one arrives at the one-loop effective action, here written in the Landau gauge

$$
\begin{aligned}
\Gamma_{\text {on-shell }}= & \frac{24 \pi F_{0}}{G R_{0}^{2}}+\frac{1}{2} \log \operatorname{det}\left[\ell^{2}\left(-\Delta_{2}+\frac{R_{0}}{6}\right)\right] \\
& -\frac{1}{2} \log \operatorname{det}\left[\ell^{2}\left(-\Delta_{1}-\frac{R_{0}}{4}\right)\right] \\
& +\frac{1}{2} \log \operatorname{det}\left[\ell^{2}\left(-\Delta_{0}-\frac{R_{0}}{3}+\frac{2 F_{0}}{3 R_{0} F_{0}^{\prime \prime}}\right)\right] .
\end{aligned}
$$

The last term is absent in the Einstein theory. As a result, in the scalar sector, one has an effective mass $M^{2}=\frac{1}{3}\left(\frac{2 F_{0}}{R_{0} F_{0}^{\prime \prime}}-R_{0}\right)$. Stability requires $M^{2}>0$, in agreement with the previous Scalaron analysis, and with the inhomogeneous perturbation analysis [7]. 


\section{Dynamical system approach}

This approach has been used by many authors [11, 3, 12, 13, 14]. One works in a cosmological setting, namely with a FRW metric, and the main idea consists in rewriting the generalized Einstein-Friedman equations in an equivalent system of first order differential equations, introducing new dynamical variables $\Omega_{i}$

$$
\frac{d}{d t} \vec{\Omega}(t)=\vec{v}(\vec{\Omega}(t))
$$

Here the evolution parameter has been denoted by $t$. The critical (or fixed ) points are defined by $\vec{v}\left(\vec{\Omega}_{0}\right)=0$. The key point is:

Hartman-Grobman theorem: The orbit structure of a dynamical system in the neighbourhood of a hyperbolic fixed point is topologically equivalent to the orbit structure of the associated linearized dynamical system, defined by a stability matrix $M_{0}$.

Recall that a hyperbolic fixed point is such that its stability matrix $M_{0}$ does not have vanishing eigenvalues. In other words the theorem states that the flux of a dynamical system in a neighbourhood of a hyperbolic fixed point can be continuously deformed to the flux of the related linearization. As a result, in order to study the stability of the above non linear system of differential Eqs. at critical points, it is sufficient to investigate the related linear system of differential Eqs.:

$$
\frac{d}{d t} \delta \vec{\Omega}(t)=M_{0} \delta \vec{\Omega}(t), \quad M_{0} \quad \text { Jacobian matrix } \quad \text { evaluated at } \vec{\Omega}_{0}
$$

The solution of the linearization is well known and the evolution is determined by the signs of the eigenvalues of $M_{0}$. As a result, the non linear system is stable if all eigenvalues of the matrix $M_{0}$ have negative real parts.

Let us apply this method to study the stability for $F(R)$ models. Introducing new variables are defined by

$$
\Omega_{R}=\frac{R}{6 H^{2}}, \quad \Omega_{F}=-\frac{f(R)-R f^{\prime}(R)}{6 H^{2}\left(1+f^{\prime}(R)\right)}, \quad \Omega_{\rho}=\frac{\chi \rho}{3 H^{2}\left(1+f^{\prime}(R)\right)},
$$

the dynamical system equivalent to Einstein-Friedman Eqs. reads

$$
\begin{aligned}
\frac{d}{d \alpha} \Omega_{R} & =2 \Omega_{R}\left(2-\Omega_{R}\right) \Omega_{R}-\beta\left(1-\Omega_{F}-\Omega_{\rho}\right) \\
\frac{d}{d \alpha} \Omega_{F} & =2 \Omega_{F}\left(2-\Omega_{R}\right)+\left(\Omega_{F}-\Omega_{R}\right)\left(1-\Omega_{F}-\Omega_{\rho}\right) \\
\frac{d}{d \alpha} \Omega_{\rho} & =\left[2\left(2-\Omega_{R}\right)-3(w+1)+1-\Omega_{F}-\Omega_{\rho}\right] \Omega_{\rho}
\end{aligned}
$$

here the evolution parameter is $\alpha(t)=\ln a(t)$ and $w=\frac{p}{\rho}$, and the function $\beta$ is $\beta(R)=$ $\frac{1+f^{\prime}(R)}{R f^{\prime \prime}(R)}$. Note that one has a complete autonomous system as soon as the quantity $\beta$ can be expressed as a function of $\Omega_{i}$. This requires the inversion of $\frac{R f^{\prime}(R)-f(R)}{R\left(1+f^{\prime}(R)\right.}=\frac{\Omega_{F}}{\Omega_{i}}$. After 
this inversion, in principle, one has $\beta=\beta\left(\Omega_{R}, \Omega_{F}\right)$, and may close the above system. The possible problems are: non unique inversions, non trivial domains with divergent points, ECT. The non linear algebraic system for critical points is

$$
\begin{aligned}
& \left.0=2 \Omega_{R}\left(2-\Omega_{R}\right) \Omega_{R}\right)-\beta\left(1-\Omega_{F}-\Omega_{\rho}\right) \\
& 0=2 \Omega_{F}\left(2-\Omega_{R}\right)+\left(\Omega_{F}-\Omega_{R}\right)\left(1-\Omega_{F}-\Omega_{\rho}\right) \\
& 0=\left[2\left(2-\Omega_{R}\right)-3(w+1)+1-\Omega_{F}-\Omega_{\rho}\right] \Omega_{\rho} .
\end{aligned}
$$

In vacuum $\rho=0$, namely $\Omega_{\rho}=0$, and de Sitter critical point existence condition follows from the solution $\Omega_{R}=2, \Omega_{F}=1$, namely $R_{0}=12 H_{0}$ and $R_{0}=R_{0} f^{\prime}\left(R_{0}\right)-2 f\left(R_{0}\right)$, in agreement with the other methods. In order to investigate the stability of this dS critical point $(2,1,0)$ the associated linear system is

$$
\begin{aligned}
\frac{d}{d \alpha} \delta \Omega_{R} & =-4 \delta \Omega_{R}+2 \beta_{0} \delta \Omega_{F}+2 \beta_{0} \delta \Omega_{\rho} \\
\frac{d}{d \alpha} \delta \Omega_{F} & =-2 \delta \Omega_{R}+\delta \Omega_{F}+\delta \Omega_{\rho} \\
\frac{d}{d \alpha} \delta \Omega_{\rho} & =0 \delta \Omega_{R}+0 \delta \Omega_{F}-3 \gamma \delta \Omega_{\rho}
\end{aligned}
$$

and one can read off the stability matrix $M_{0}$, whose eigenvalues are $\lambda_{1}=-3 \gamma, \quad \gamma>0$ and

$$
\lambda_{2,3}=\frac{1}{2}\left(-3 \pm \sqrt{25-16 \beta_{0}}\right)
$$

The stability condition associated with the de Sitter critical point requires that the real part of all eigenvalues has to be negative, thus

$$
\frac{1+f^{\prime}\left(R_{0}\right)}{R_{0} f^{\prime \prime}\left(R_{0}\right)}>1
$$

again in agreement with Scalaron perturbation analysis and one-loop de Sitter calculation. In the matter-radiation sector, where $\Omega_{\rho}$ is non vanishing, other critical points, in general, exist, but their analytical determination, in realistic cases, is problematic, since one has to know explicitly $\beta$ in order to close the system, and numerical analysis, in general, is necessary.

We conclude recalling that, within this approach, it is not difficult to deal with generalizations of the kind $F(R) \longrightarrow F(R, G, Q, .$.$) , [15] where F$ depends on arbitrary invariants of tensor curvature as $G$, Gauss-Bonnet invariant, $Q$ the square of Riemann tensor, and so on. In the case $F(R, G, Q)$, the associated de Sitter existence solution reads $F=\frac{R F_{R}^{\prime}}{2}-\frac{R^{2}}{6}\left(F_{G}^{\prime}+F_{Q}^{\prime}\right)$ and the related stability condition is (see [16] and references therein)

$$
\frac{F_{R}^{\prime}+\frac{2}{3} R F_{Q}^{\prime}}{R\left[F_{R R}^{\prime \prime}+\frac{2}{3} F_{Q}^{\prime}+\frac{2}{3} R\left(F_{G R}^{\prime \prime}+F_{R Q}^{\prime \prime}\right)+\frac{1}{9} R^{2}\left(F_{G G}^{\prime \prime}+2 F_{G Q}^{\prime \prime}+F_{Q Q}^{\prime \prime}\right)\right]}>1
$$




\section{CONCLUDING REMARKS}

Modified gravity may be seen as the phenomenological description of a fundamental unknown theory. From this point of view, corrections to Einstein-Hilbert action depending on higher order curvature invariants are likely to be expected (Lovelock gravity is an example).

Among many existing approaches, three methods have been illustrated in order to investigate the stability of these models around de Sitter critical points, and the dS stability conditions has been derived in all the three approaches.

These methods have owns advantages and problems, but, in our opinion, the third one, the dynamical system approach, permits to study critical points and stability for modified gravitational models depending on arbitrary geometric invariants, generalising the results obtained for $F(R)$ models.

\section{ACKNOWLEDGMENTS}

Thanks to G. Cognola, E. Elizalde, S. Nojiri, S. D. Odintsov and L. Vanzo useful for discussions.

\section{REFERENCES}

1. G. R. Dvali, G. Gabadadze and M. Porrati, Phys. Lett. B 485, 208-214 (2000).

2. E. Copeland, M. Sami and S. Tsujikawa, Int. J. Mod. Phys. D 15 1753-1936 (2006); S. Nojiri, S. D. Odintsov, Int. J. Geom. Meth. Mod. Phys. 4, 115-146 (2007).

3. A. A. Starobinsky, Phys. Lett. B 91, 99-146 (1980).

4. S. Capozziello, S. Carloni and A. Troisi, Recent Res. Dev. Astron. Astrophys. 1, 625- (2003); S. M. Carroll, V. Duvvuri, M. Trodden and M. S. Turner, Phys. Rev. D 70, 043528 (2004).

5. I. Sawicki and W. Hu, Phys. Rev. D 76,064004 (2007); A. A. Starobinsky, JETP Lett. 86, 157-163 (2007); S. Nojiri and S. D. Odintsov, Phys. Lett. B 657, 238-245 (2007).

6. G. Cognola, E. Elizalde, S. Nojiri, S. D. Odintsov, L. Sebastiani and S. Zerbini,Phys. Rev. D 77, 046009 (2008).

7. V. Faraoni, Phys. Rev. D 72, 124005 (2005).

8. E. S. Fradkin, and A. A. Tseytlin, Nucl. Phys. B 234, 472-508 (1984).

9. G. Cognola, E. Elizalde, S. Nojiri, S. D. Odintsov and S. Zerbini, JCAP 0502, 010 (2005); G. Cognola and S. Zerbini, J. Phys. A 39, 6245-6251 (2006).

10. E. Elizalde, L. Vanzo and S. Zerbini, Comm. Math. Phys. 194, 613-630 (1998).

11. V. T. Gurovich and A. A. Starobinsky, Sov. Phys. JEPT 50 , 844-852 (1979).

12. G. E. R. Ellis and J. Wainwright Editors, Dynamical System in Cosmology, Cambridge University Press, Cambridge (1997).

13. L. Amendola, R. Gannouji, D. Polarski and S. Tsujikawa, Phys. Rev. D 75, 083504 (2007).

14. S. Carloni, P. K. S. Dunsby, S. Capozziello and A. Troisi, Class. Quant. Grav. 22, 4839-4868 (2005); S. Carloni, A. Troisi and P. K. S. Dunsby, Some remarks on the dynamical systems approach to fourth order gravity, arXiv:0706.0452 [gr-qc].

15. S. M. Carroll, A. De Felice, V. Duvvuri, D. A. Easson, M. Trodden and M. S. Turner, Phys. Rev. D 71, 063513 (2005).

16. G. Cognola, M. Gastaldi and S. Zerbini, Int. J. Theor. Phys. 47, 898-910 (2008); G. Cognola and S. Zerbini, Homogeneous cosmologies in generalized modified gravity, arXiv:0802.3967 [hep-th]. 